\title{
Far-Ultraviolet Observations of RR Lyrae Stars in the Core of NGC $1851^{1}$
}

\author{
Ronald A. Downes ${ }^{2}$, Bruce Margon ${ }^{2}$, Lee Homer ${ }^{3}$, and Scott F. Anderson ${ }^{3}$
}

\begin{abstract}
There are extraordinarily few far ultraviolet observations of RR Lyrae stars in the literature. We present Hubble Space Telescope FUV $(\sim 1600 \AA)$ imaging of the core of the globular cluster NGC 1851. Eleven new variables whose light curves are consistent with those of RR Lyr stars are discovered, increasing the total number of RR Lyr known in this cluster by a substantial amount. In agreement with basic physical theory, the observed amplitude of the variables in the FUV is enormous compared with the century of past optical observations, ranging up to 4 mag. HST STIS FUV observations of cluster cores may prove an especially effective means of obtaining a near-complete census of RR Lyr stars, combining high angular resolution, suppression of luminous red stars, and data where the stellar pulsation amplitudes are greatly enhanced. Attention is also drawn to a peculiar blue object in the cluster that is most probably a low mass x-ray binary system in quiescence.
\end{abstract}

Subject headings: globular clusters: individual (NGC 1851), RR Lyrae variables

\section{Introduction}

A complete census of RR Lyrae stars in a globular cluster is important, as it is the basis for determining the Oosterhoff type of the cluster, which in turn is used to sort various cluster properties. Hogg (1973) first identified 10 variables in NGC 1851, while Liller (1975) classified and obtained periods for those objects, plus an additional four variables. Eleven of the 14 variables were identified as RR Lyr types, and based on the mean period of the RR $a b$ ( $<0.6$ days), Liller (1975) classified the cluster as Oosterhoff Type I. Wehlau et al. (1978) further refined the periods for the twelve previously known objects (one of the variables for which Liller (1975) failed to detect any variations is in fact an RR Lyr star), as well as determined the periods of an additional

\footnotetext{
${ }^{2}$ Space Telescope Science Institute, 3700 San Martin Drive, Baltimore, MD 21218; downes@stsci.edu, margon@stsci.edu

${ }^{3}$ Department of Astronomy, University of Washington, Box 351580, Seattle, WA 98195-1580; anderson@astro.washington.edu, homer@astro.washington.edu

${ }^{1}$ Based on observations made with the NASA/ESA Hubble Space Telescope, obtained at the Space Telescope Science Institute, which is operated by the Association of Universities for Research in Astronomy, Inc., under NASA contract NAS 5-26555.
} 
seven objects. Those authors found the mean period of the RR $a b$ was 0.573 days, consistent with Liller (1975), and the ratio of $\mathrm{RR} c: \mathrm{RR} a b=0.36$. Thus, NGC 1851 was at the extreme end of the Oosterhoff Type I clusters listed in Wehlau \& Demers (1977).

Wehlau et al. (1982) discovered three more RR Lyr variables, raising the total to 22 objects, and found that the mean period of the RR $a b$ was now 0.572 days. However, the ratio of RR $c: \operatorname{RR} a b$ jumped dramatically from 0.36 to 0.47 , again putting NGC 1851 at the extreme end of Oosterhoff Type I clusters. More recently, Walker (1998) performed a thorough search of NGC 1851 for RR Lyr stars in the optical, and found seven more variables for a total of 29 . The mean period of the RRab increased slightly to 0.586 days, but the ratio of $\mathrm{RR} c$ :RR $a b$ was reduced back to 0.38 . Castellani, Caputo, \& Castellani (2003) list basic data on globular clusters with at least 10 RR $a b$ and $5 \mathrm{RR} c$ variables, and NGC 1851 has the most extreme mean period of the RRab, while the ratio $\mathrm{RR} c: \mathrm{RR} a b$ is high but not extreme. However, if the census of RR Lyr stars is incomplete, the true standing of the cluster with respect to other Oosterhoff Type I clusters (which may also be incomplete) is uncertain.

As noted by Walker (1998), RR Lyr stars in the central region of the cluster, which is too crowded for ground-based observations to resolve, are certainly expected. Recently Sumerel et al. (2004) have reported 19 further variables in the cluster, some quite close to the core, using ground-based data, and they conclude that most are RR Lyr stars. Also, Saviane et al. (1998) used pre-COSTAR Hubble Space Telescope (HST) WF/PC data to identify 7 new RR Lyr candidates. It is clear that HST observations of the cluster (particularly with instruments that correct for spherical abberation) are capable of adding important new data on variables in the cluster center.

We obtained far-ultraviolet imaging observations of NGC 1851 with the HST STIS to search for variability of the x-ray burster located in the cluster core. While that candidate object (Deutsch et al. 1996) was not found to be variable (Homer et al. 2001), several other objects are easily seen (by visual inspection alone) to be highly variable, with amplitudes of 2-4 magnitudes! We therefore measured the light curves of all objects in the cluster to identify all large-amplitude variables, and examination of these light curves reveal that the large-amplitude variables are very likely RR Lyr stars. Thus, we may now have a near-complete census of the NGC 1851 RR Lyr population, which will allow us to determine if it is a typical or atypical Oosterhoff Type I cluster.

\section{Observations and Analysis}

On 1999 March 24, we obtained 4 orbits of HST STIS FUV-MAMA imaging data with the long-pass quartz (F25QTZ) filter. This filter has a central wavelength of $1595 \AA$, and a FWHM of $230 \AA$. The data were obtained in time-tag mode to support the temporal analysis desired for the main goal of the program - a study of the x-ray burster in the core of the cluster (Homer et al. 2001). A thorough analysis of the data reveals 11 objects that were significantly variable, and basic information of these stars is given in Table 1. Figure 1 shows two of the STIS images, 
with the variables identified. To better illustrate the dramatic nature of the variations, we show in Figure 2 the full range for the four largest amplitude variables.

On 1996 April 10, we obtained HST WFPC2 images of NGC 1851 through the F439W and F336W filters; these filters are very similar to the Johnson B and U, respectively. To aid in identifying the objects for future studies, we include in Figure 1 the F439W image. Our prior photometric analysis (Deutsch et al. 1996) determined the WFPC2 magnitudes for 7 of the variables, and these data are included in Table 1; the four missing objects include one not on the PC image, and three that are in regions too crowded to obtain accurate measurements.

Prediscovery photometry of these variables from $H S T$ is also available in the $\mathrm{F} 439 \mathrm{~W}$ and F555W filters, approximating $B$ and $V$, from the work of Piotto et al. (2002). We also include these colors in Table 1. It is immediately apparent that all of these stars lie in the region of the cluster color-magnitude diagram occupied by RR Lyr stars. Although we have insufficient data to derive mean magnitudes, all of the candidate objects have $0<(B-V)<0.5$, in common with the well-studied RR Lyr's in NGC1851 discussed by Walker (1998). All of our candidates also lie within 0.8 mag of the mean $V$ of the cluster RR Lyr's observed by Walker (1998), suggesting that the luminosity of the HST variables is also consistent with this classification, although better observations are surely desirable.

The light curves for the variables are shown in Figure 3, which reveals that the objects are indeed most likely RR Lyr variables. Even though we do not cover a full period for any of the objects, the distinctive shape of the light curves allows us to tentatively classify most of the objects. Note that IUE observations of the RRab stars RR Lyr and X Ari (Bonnell \& Bell 1985), and the RRc star DH Peg (Fernley et al. 1990), show that the light curve shapes seen in the visible are reproduced in the ultraviolet, so FUV light curve shape can still be used to classify the stars. One exception may be RR08, for which a period of $\sim 160$ minutes may be appropriate, thus ruling out an RR Lyr classification. It is interesting to note that of the $\sim 160$ objects detected in the STIS far-ultraviolet (FUV) image, $7 \%$ are RR Lyr stars. Given this high fraction, and the extremely large amplitude, it appears that the FUV may be the best place to search for RR Lyr variables.

We have unsuccessfully attempted to correlate the objects discussed by Sumerel et al. (2004) with the variables observed by HST. Nine of their objects fall in the region covered by our data, but only six of these have plausible counterparts in WFPC2 data (even allowing for reference frame offsets). Further, of those six, three are far too faint to correspond to RR Lyr stars in the cluster, and none appear in our FUV data, quite contrary to our expectations. We do note that many of the Sumerel et al. (2004) candidates fall very near, but not coincident with, multiple variables found in the HST data, leading to the possibility that some of the ground-based variables are superpositions of multiple variable stars separated by $\sim 1^{\prime \prime}$. Also note that none of the WF/PC candidates of Saviane et al. (1998) fall in our STIS field, and that Sumeral object NV17 is coincident with Saviane object 1 (although the classifications differ).

A search of the HST archive reveals that there is only one other long duration, FUV imaging 
observation of a globular cluster that could be used to search for RR Lyr stars. Analysis of this dataset on NGC 6752 (obtained by M. Shara on 2001 March 30) revealed no RR Lyr stars, despite the fact that the objects should be brighter than those in NGC 1851, as the former cluster is $\sim 2$ mag closer. A search of the literature shows that no RR Lyr stars have been detected in the optical for this cluster (Clement et al. 2001), so it is perhaps not surprising that none were detected in the FUV.

\section{Discussion}

\subsection{RR Lyrae Stars}

In the optical, RR Lyr stars have a typical amplitude of 0.5-1.0 mag, whereas our ultraviolet data shows a larger 2-4 mag variation. Ultraviolet observations from the ANS satellite of RR Lyr itself (Bonnell et al. 1982) show that the amplitude of the pulsations steadily increases toward the far-ultraviolet, going from $0.8 \mathrm{mag}$ at $3300 \AA$ to $3.1 \mathrm{mag}$ at $1800 \AA$. As a first approximation, the amplitude of the pulsation should vary as $(\mathrm{dB} / \mathrm{dT}) / \mathrm{B}$, where $\mathrm{B}$ is the Planck function and $\mathrm{T}$ is the temperature. Of course, this model is accurate only if the pulsation is non-radial, which is not the case for RR Lyr stars. However, if the radial variations are not a function of wavelength, then this simplified model will be a reasonable approximation for the wavelength dependence of the amplitude.

For RR Lyr itself $(\mathrm{T} \sim 7000 \mathrm{~K})$, we find the ratio of the amplitudes at $1800 \AA$ and $5500 \AA$ to be 3.4 , so for a $\sim 1$ mag amplitude at $5500 \AA$, this simple model would predict an $1800 \AA$ amplitude of

$3.4 \mathrm{mag}$, very close to what is observed. For our more ultraviolet wavelength $(1400 \AA)$, we derive an amplitude of 4.6 mag. We show, in Table 2, the FUV amplitudes for several temperatures for two different $\mathrm{V}$ amplitudes. The ultraviolet amplitudes of the NGC $1851 \mathrm{RR}$ Lyr stars fit nicely in these ranges.

If we consider only the well-studied objects outside the cluster core, there were 29 previously known RR Lyr variables, so with the 11 new candidates presented here, the total count is now 40 variables. While we do not have periods for the new objects, we have determined their variability classes. As previously noted, Wehlau et al. (1978) found the ratio RRc:RRab to be 0.36, Wehlau et al. (1982) increased the value to 0.47, and Walker (1998) reduced the value back to 0.38. Our updated value for the ratio is 0.54 , which is the second highest of all Oosterhoff Type I clusters (Castellani, Caputo, \& Castellani 2003). If the Saviane et al. (1998) candidates are included, the ratio drops back to 0.47. We do not include the candidates of Sumerel et al. (2004) because of the concerns previously noted. When coupled with the fact that NGC 1851 has the most extreme mean period of the RRab, this cluster is truly an unusual Oosterhoff Type I object. 


\subsection{Other Interesting Objects}

In addition to looking for variable objects in the data, we combined the STIS and WFPC2 data to search for UV-bright objects. As noted by Deutsch et al. (1996), the WFPC2 data revealed four UV-bright objects, two of which are also in the STIS image; both objects are marked in Figure 1 on the STIS images. A WFPC2 chart for the x-ray burster can be found in Deutsch et al. (1996), while Figure 4 shows a finding chart for the other UV-bright object. Data for these objects - the $\mathrm{x}$-ray burster (XRB) and the other UV-bright star (UV) - are given in Table 3. Neither object is variable, while the UV-bright star is significantly bluer in the far ultraviolet than the x-ray burster.

Reanalysis of the Chandra HRC-S zeroth order image of NGC 1851 (Homer et al. 2001) finds a possible faint X-ray source positionally coincident with the UV-bright object. At $\mathrm{L}_{x} \sim 10^{33}$ $\operatorname{ergs~s}^{-1}$, this would suggest a quiescent low-mass x-ray binary (qLMXB). However, its optical/UV brightness $\left(\mathrm{M}_{V} \sim 3\right)$ implies a much lower $\mathrm{L}_{x} / \mathrm{L}_{\text {opt }}$ ratio than the optically identified (in quiescence) qLMXB X5 in 47 Tucanae $\left(\mathrm{L}_{x}=9.2 \times 10^{32} \mathrm{ergs} \mathrm{s}^{-1}\right.$ and $\mathrm{M}_{V}=8.2$; Edmonds et al. (2002)) Of course, since the Chandra and HST observations were taken nine months apart, it is possible that the object was in an active state during the $H S T$ observations and a quiescent state during the Chandra observations, which would skew the $\mathrm{L}_{x} / \mathrm{L}_{\text {opt }}$ ratio.

\section{Summary}

We have presented one of the few FUV observations of a globular cluster core, and find that RR Lyr stars are surprising numerous and prominent in NGC 1851. FUV observations of cluster cores may prove an especially effective means of obtaining a near-complete census of RR Lyr stars, combining high angular resolution, suppression of luminous red stars, and data where the stellar pulsation amplitudes are greatly enhanced.

We thank E. Deutsch for providing WFPC2 photometry, and Mike Corwin for providing coordinate information necessary to correlate the Sumerel et al. (2004) data with the WFPC2 data. Alister Walker provided helpful comments on an early draft of this paper, and Howard Bond provided software to analyze the wavelength dependence of the pulsation amplitude. 


\section{REFERENCES}

Bonnell, J.T. \& Bell, R.A. 1985, PASP, 97, 236

Bonnell, J., Wu, C.-C., Bell, R.A., \& Hutchinson, J.L. 1982, PASP, 94, 910

Castellani, M., Caputo, F., \& Castellani, V. 2003, A\&A, 410, 871

Clement, C.M., Muzzin, A., Dufton, Q., Ponnampalam, T., Wang, J., Burford, J., Richardson, A., \& Rosebery, T. 2001, AJ, 122, 2587

Deutsch, E.W., Anderson, S.F., Margon, B., \& Downes, R.A. 1996, ApJ, 472, L97

Edmonds, P.D., Heinke, C.O., Grindlay, J.E., and Gilliland, R.L. 2002, ApJ, 564, L17

Fernley, J.A., Skillen, I., Jameson, R.F., \& Longmore, A.J. 1990, MNRAS, 242, 685

Hogg, H.S. 1973, Pub. David Dunlap Obs., 3, No. 6

Homer, L., Anderson, S.F., Margon, B., Deutsch, E.W., \& Downes, R.A. 2001, ApJ, 550, L155

Liller, M.H. 1975, ApJ, 201, L125

Piotto, G. et al. 2002, A\&A, 391, 945

Saviane, L., Piotto, G., Fagotto, F., Zaggia, S., Capaccioli, M., \& Aparicio, A. 1998, A\&A, 333, 479

Sumerel, A. N., Corwin, T. M., Catelan, M., Borissova, J., \& Smith, H. A. 2004, IBVS 5533

Walker, A. 1998, AJ, 116, 220

Wehlau, A. \& Demers, S. 1977, A\&A, 57, 251

Wehlau, A., Liller, M.H., Clement, C.C., \& Wizinowich, P. 1982, AJ, 87, 1295

Wehlau, A., Liller, M.H., Demers, S., \& Clement, C.C. 1978, AJ, 83, 598 
Table 1. RR Lyrae Candidates in NGC 1851

\begin{tabular}{|c|c|c|c|c|c|c|c|}
\hline Name & R.A. $(2000)^{a}$ & Decl. $(2000)^{\mathrm{a}}$ & $m_{555^{\mathrm{b}}}$ & $m_{439}-m_{555}{ }^{\mathrm{c}}$ & $m_{336}-m_{439} \mathrm{~d}$ & Range $\left(m_{q t z}\right)^{\mathrm{e}}$ & Type \\
\hline $\mathrm{RR} 1$ & 051407.057 & -400254.99 & 16.09 & 0.38 & 0.78 & 4.3 & $\mathrm{RR} a b$ \\
\hline RR2 & 051406.709 & -400256.45 & 16.40 & 0.42 & & 2.6 & $\mathrm{RR} a b$ \\
\hline RR3 & 051407.205 & -400247.64 & 16.41 & 0.44 & 0.91 & 2.9 & $\mathrm{RR} a b$ \\
\hline $\mathrm{RR} 4$ & 051406.185 & -400254.24 & 16.23 & 0.31 & 1.13 & 1.7 & $\mathrm{RR} c$ \\
\hline RR5 & 051407.147 & -400245.94 & 16.29 & 0.21 & 0.89 & 2.8 & $\mathrm{RR} c$ \\
\hline RR6 & 051407.604 & -400241.79 & & & & 2.0 & $\mathrm{RR} c$ \\
\hline RR7 & 051406.548 & -400250.05 & 15.19 & 0.06 & 0.35 & 3.3 & $\mathrm{RR} c$ \\
\hline RR8 & 051406.140 & -400248.28 & 16.81 & 0.34 & 1.10 & 1.3 & RRc:: \\
\hline RR9 & 051406.500 & -400243.43 & 16.19 & 0.22 & & 1.6 & $\mathrm{RR} c:$ \\
\hline RR10 & 051406.476 & -400242.59 & 15.42 & 0.13 & & 3.2 & $\mathrm{RR} a b$ \\
\hline RR11 & 051406.208 & -400244.61 & 16.09 & 0.17 & 0.89 & 2.4 & $\mathrm{RR} a b$ \\
\hline
\end{tabular}

a Units of right ascension are hours, minutes, and seconds, and units of declination are degrees, arcminutes, and arcseconds. Coordinates measured on STIS FUV-MAMA image 04wm02euq.

b WFPC2 F555W filter

c WFPC2 F439W and F555W filters. Note that all exposures were obtained within a 15 minute interval, so variability of the objects should not significantly distort the color.

d WFPC2 F336W and F439W filters. Note that the F336W and F439W exposures were obtained 80 minutes apart, so variability of the objects could distort the color. Also, this color was obtained at a different time from the other WFPC2 values in this table.

e STIS F25QTZ filter 
Table 2. Predicted Ultraviolet (1500Å) Amplitudes for RR Lyrae Stars

\begin{tabular}{rcc}
\hline \hline $\begin{array}{c}\text { Temperature } \\
(\mathrm{K})\end{array}$ & $\begin{array}{c}\text { UV Amplitude } \\
(\mathrm{V} \text { amp. }=0.5)\end{array}$ & $\begin{array}{c}\text { UV Amplitude } \\
(\mathrm{V} \text { amp. }=1.0)\end{array}$ \\
\hline 7000 & 2.3 & 4.6 \\
8000 & 1.9 & 3.8 \\
9000 & 1.7 & 3.3 \\
10000 & 1.4 & 2.8 \\
\hline
\end{tabular}


Table 3. UV-bright Objects in NGC 1851

\begin{tabular}{rrrrrrr}
\hline \hline Name & R.A. $(2000)^{\mathrm{a}}$ & Decl. $(2000)^{\mathrm{a}}$ & $m_{439}{ }^{\mathrm{b}}$ & $m_{336}-m_{439} \mathrm{~b}^{\mathrm{b}, c}$ & $m_{q t z}{ }^{\mathrm{d}}$ & $m_{q t z}-m_{336}$ \\
\hline $\mathrm{XRB}^{\mathrm{e}}$ & 051406.469 & -400239.01 & 20.46 & -0.74 & 18.2 & -1.52 \\
$\mathrm{UV}^{\mathrm{f}}$ & 051406.853 & -400249.96 & 18.96 & -0.78 & 16.1 & -2.08 \\
\hline
\end{tabular}

a Units of right ascension are hours, minutes, and seconds, and units of declination are degrees, arcminutes, and arcseconds. Coordinates measured on STIS FUV-MAMA image 04wm02euq.

b WFPC2 F439W filter

c WFPC2 F336W filter

d STIS F25QTZ filter

e X-ray burster, Deutsch et al (1996)

f UV-excess object, this work; corresponds to a faint x-ray source (see text) 

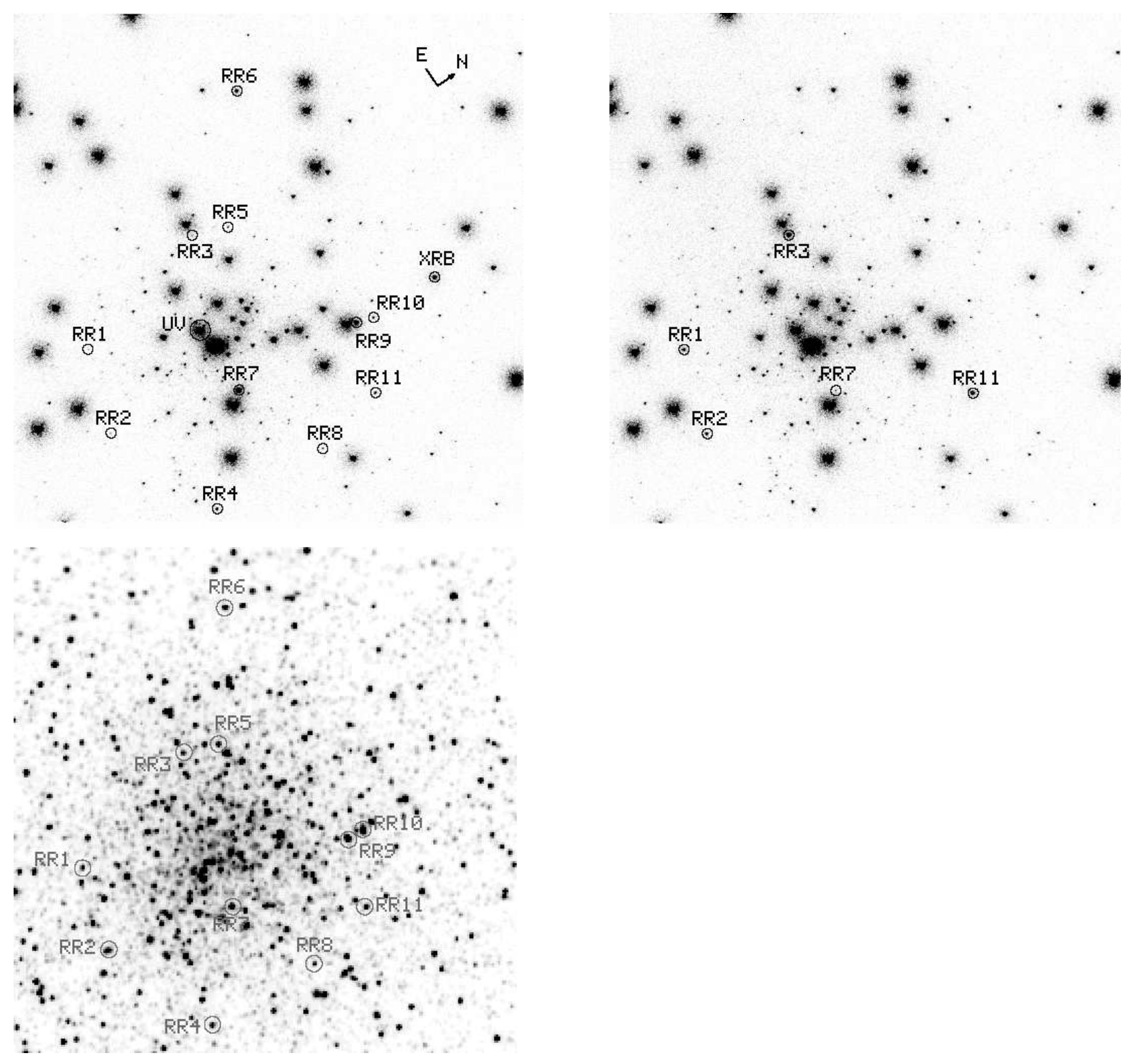

Fig. 1. - Top panels: $25^{\prime \prime} \times 25^{\prime \prime} H S T$ STIS FUV-MAMA images of the core of NGC 1851 with the F25QTZ filter. The candidate RR Lyrae stars are marked, as are two other interesting objects (see text for details). Objects RR1, RR2, RR3, and RR11 are at maximum on the right image, while RR7 is at maximum on the left image. Bottom panel: the same field with the HST WFPC2 PC and the F439W filter. 

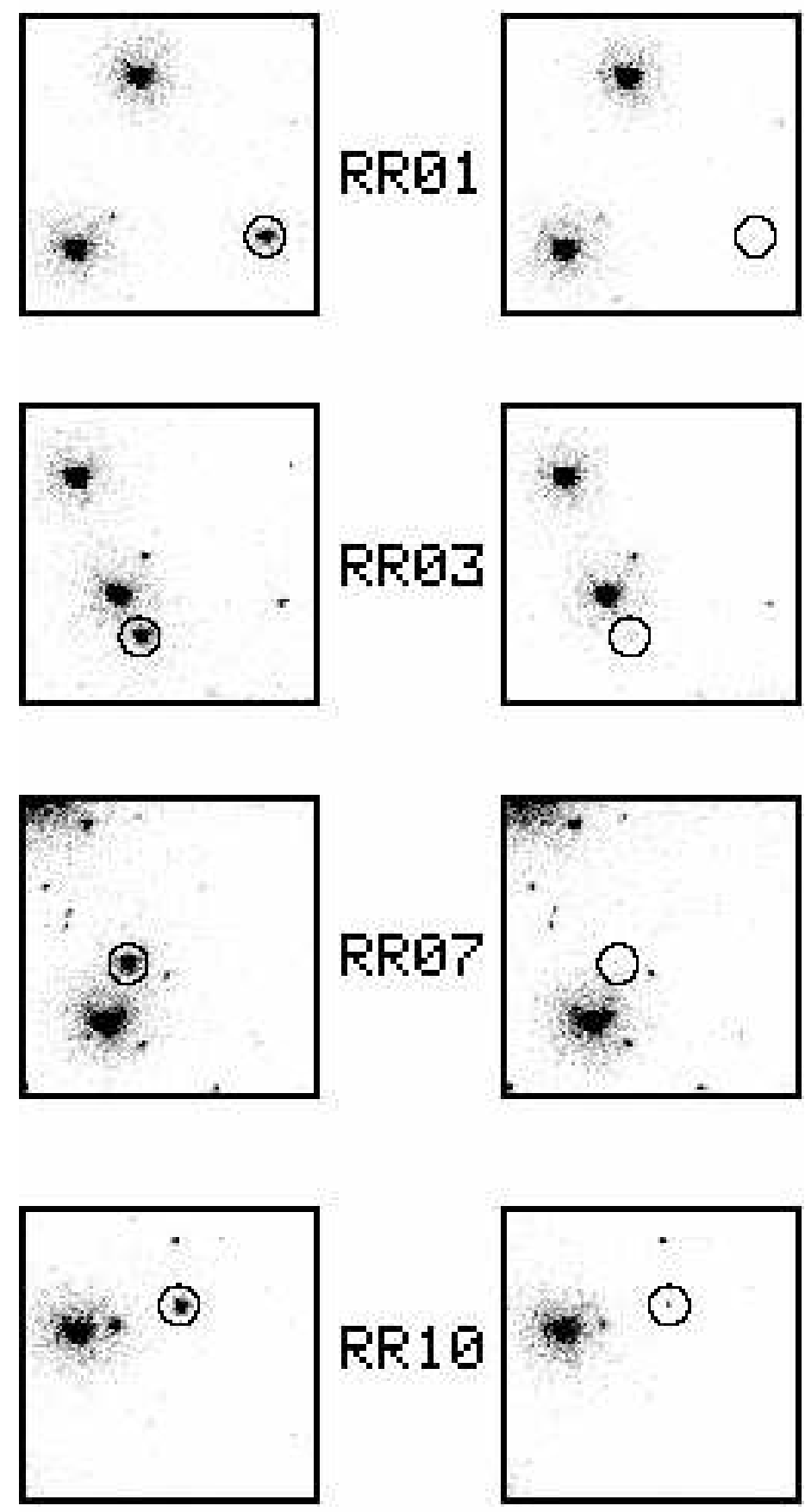

Fig. 2.- Observations at maximum (left) and minimum (right) for a sample of the RR Lyr stars. These images demonstrate the how straightforward it is to detect these variables in the far-ultraviolet. 

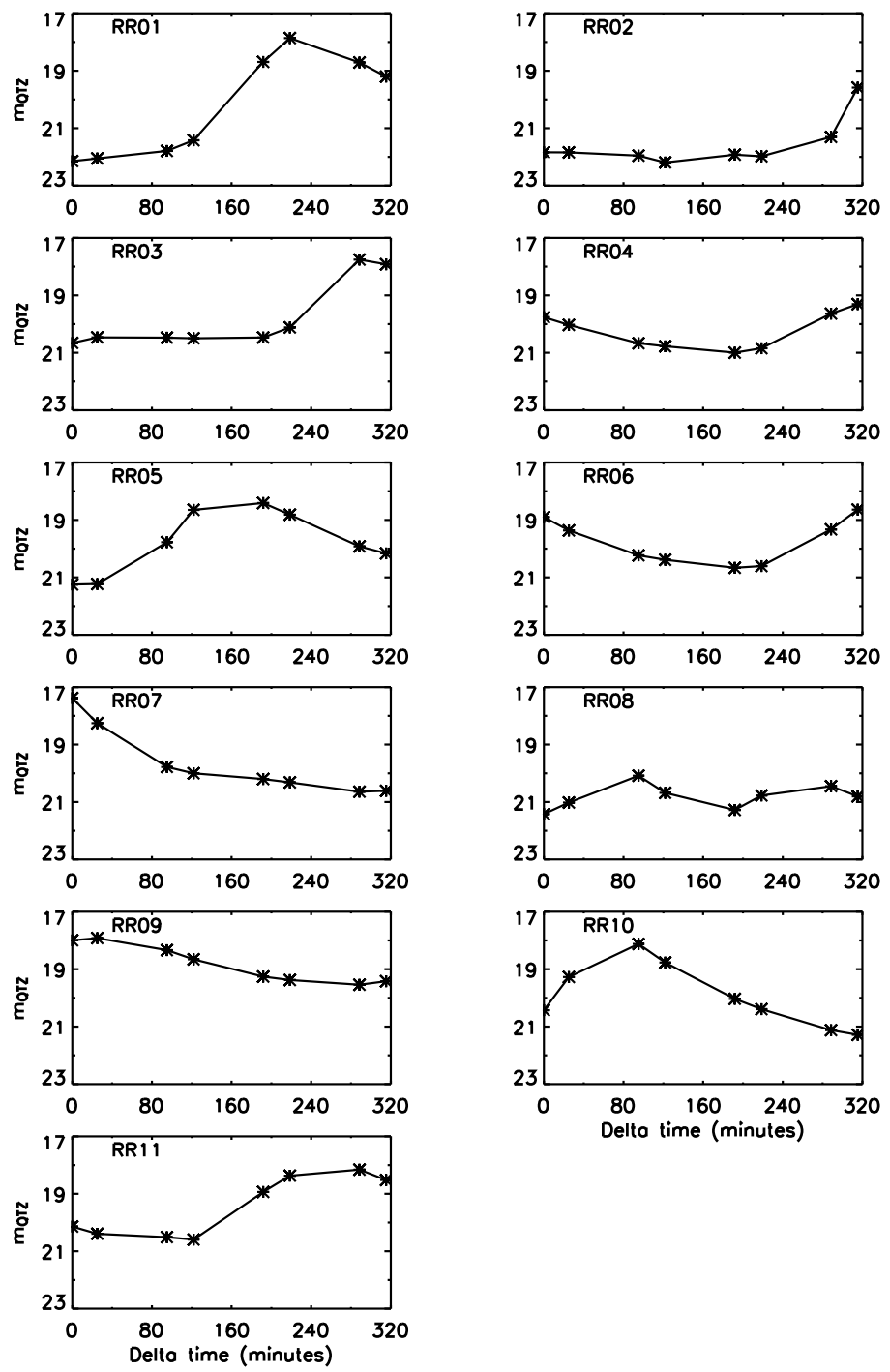

Fig. 3. - Light curves for the 11 RR Lyrae candidates. 


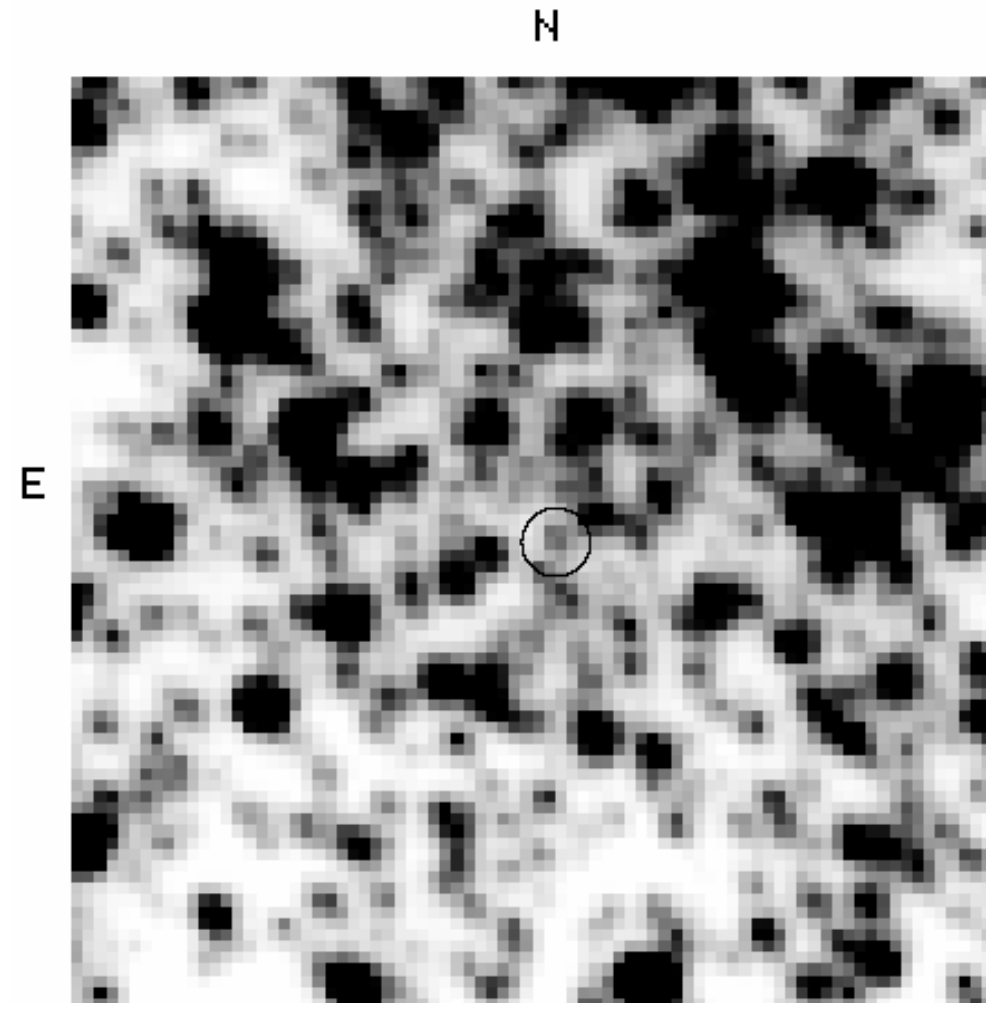

Fig. 4. $-2^{\prime \prime} \times 2^{\prime \prime}$ HST WFPC2 image of the UV-excess object with the F439W filter. 